University of Nebraska - Lincoln

DigitalCommons@University of Nebraska - Lincoln

John R. Hardy Papers

Research Papers in Physics and Astronomy

2-1997

\title{
Centimeter-Wave Reflection in the Nitrates and Nitrites of Sodium and Potassium: Experiment and Theory
}

Steven G. Cornelison

U.S. Army Research Laboratory, Aberdeen Proving Ground, Maryland

Arthur Guass, Jr.

U.S. Army Research Laboratory, Aberdeen Proving Ground, Maryland

J. Krane

University of Nebraska - Lincoln

John R. Hardy

University of Nebraska - Lincoln

Follow this and additional works at: https://digitalcommons.unl.edu/physicshardy

Part of the Physics Commons

Cornelison, Steven G.; Guass, Jr., Arthur; Krane, J.; and Hardy, John R., "Centimeter-Wave Reflection in the Nitrates and Nitrites of Sodium and Potassium: Experiment and Theory" (1997). John R. Hardy Papers. 4. https://digitalcommons.unl.edu/physicshardy/4

This Article is brought to you for free and open access by the Research Papers in Physics and Astronomy at DigitalCommons@University of Nebraska - Lincoln. It has been accepted for inclusion in John R. Hardy Papers by an authorized administrator of DigitalCommons@University of Nebraska - Lincoln. 


\title{
Centimeter-wave reflection in the nitrates and nitrites of sodium and potassium: Experiment and theory
}

\author{
Steven G. Cornelison and Arthur Gauss, Jr. \\ U.S. Army Research Laboratory, Aberdeen Proving Ground, Maryland 21005-5066 \\ J. Krane and J. R. Hardy \\ Department of Physics and Center for Electro-Optics, University of Nebraska-Lincoln, Lincoln, \\ Nebraska 68588-0111
}

(Received 11 July 1996; accepted for publication 11 October 1996)

\begin{abstract}
Temperature-dependent centimeter-wave reflection is studied in powdered samples of potassium nitrate $\left(\mathrm{KNO}_{3}\right)$, potassium nitrite $\left(\mathrm{KNO}_{2}\right)$, sodium nitrate $\left(\mathrm{NaNO}_{3}\right)$, and sodium nitrite $\left(\mathrm{NaNO}_{2}\right)$. Temperature-dependent reflection measurements at centimeter-wave frequencies were performed on an HP8510B Network analyzer based reflectometer. These measurements are compared to calculations utilizing a Debye relaxation model. Reflection losses seen in $\mathrm{KNO}_{2}$ and $\mathrm{NaNO}_{2}$ are expected to be due to the presence of permanent dipoles that are excited to "hopping" modes as the temperature is raised. Although $\mathrm{NaNO}_{3}$ shows little reflection losses, $\mathrm{KNO}_{3}$ shows significant losses as the temperature is raised toward the order/disorder transition temperature of $128{ }^{\circ} \mathrm{C}$. This is believed to be due to the development of "induced" dipole moments as the lattice becomes increasingly disordered. (C) 1997 American Institute of Physics. [S0021-8979(97)04002-4]
\end{abstract}

\section{INTRODUCTION}

The dielectric properties of materials at very high frequencies (i.e., optical frequencies) and at relatively low frequencies (megahertz or less) have been studied extensively. Fewer studies have been performed within the intermediate region of centimeter- or millimeter-wave frequencies. ${ }^{1,2}$ Here the results of centimeter-wave reflection measurements and theoretical modeling studies for potassium nitrite $\left(\mathrm{KNO}_{2}\right)$, potassium nitrate $\left(\mathrm{KNO}_{3}\right)$, sodium nitrate $\left(\mathrm{NaNO}_{3}\right)$, and sodium nitrite $\left(\mathrm{NaNO}_{2}\right)$ are reported. Experimental studies were performed from 2 to $20 \mathrm{GHz}$ in a centimeter-wave reflectometer over a temperature range from room temperature $\left(25^{\circ} \mathrm{C}\right)$ to about $250{ }^{\circ} \mathrm{C}$. The dielectric losses in these materials are expected to be mostly dipolar in the frequency range studied.

The losses in the nitrites are expected to be due to the permanent dipole moments associated with the nitrite ions, which are excited to "hopping" modes when the temperature is raised. ${ }^{1,3}$ Sodium nitrate, which contains no permanent dipole moments, shows absorptions similar to that expected for ionic salts. ${ }^{4}$ Potassium nitrate shows interesting, temperature-dependent absorption in this frequency range. This is believed to be due to a dynamically induced dipole moment, which begins to develop at temperatures just below the order/disorder transition temperature of $128^{\circ} \mathrm{C} .^{3,5,6}$

The mechanisms that underlie the dielectric behavior of these materials are discussed in the theoretical section. Reflection losses using a Debye relaxation model calculation are compared to and are consistent with temperaturedependent centimeter-wave reflection measurements on sodium nitrite, potassium nitrite, and potassium nitrate.

\section{EXPERIMENT}

Powder samples were measured using the powder sample heater as diagramed in Fig. 1. These salts were first powdered in an analytical mill, oven dried for several hours at $135^{\circ} \mathrm{C}$, and then lightly packed to a thickness of a centimeter or so immediately before the measurements were to be taken. The "lightly packed" powders had approximately $50 \%$ of theoretical density. The temperature-controlled heating element is located at the bottom of the sample holder below a 0.25 in. aluminum plate, which also served as a conducting back plane. Temperature variations over the back plane were typically less than $1{ }^{\circ} \mathrm{C}$. As the temperature is raised, the thermocouples located at various depths in the powder sample measured the temperature during the runs. The reported temperature is an average of temperatures measured at the three different depths within the sample. ${ }^{4}$ Temperature variations within the samples are generally of the order of $10^{\circ} \mathrm{C}$ or less.

An almost fully dense ( $>95 \%$ density) potassium nitrate sample was produced by pressing potassium nitrate powder (to $11 \mathrm{ksi}$ ) in a $15.2 \mathrm{~cm}^{2}$ mold to a thickness of $8.1 \mathrm{~mm}$. This sample was attached to an aluminum backing plate with conductive paste and then placed on top of the top plate of the heater. During the heating process, another aluminum plate with connected thermocouples was placed on top of the potassium nitrate sample. When the temperatures of the heater plate and the top plate were within $5{ }^{\circ} \mathrm{C}$ of each other, the top was quickly removed and the measurement taken. Because of this, the temperature measurement of the pressed powder sample was more accurate than for the loose powder samples.

The radar reflection measurements were taken from 2 to $20 \mathrm{GHz}$ using an HP8510B-based centimeter-wave reflectometer. ${ }^{4}$ This reflectometer is in the standard form of an arch with the sample resting on a pedestal surrounded by radar-absorbing material. The bistatic angle for these tests was $5^{\circ}$. During each test, the empty sample holder was first measured to obtain a base line measurement, then the sample was added for the temperature sweeps. The samples were measured through several temperature cycles, and the data 


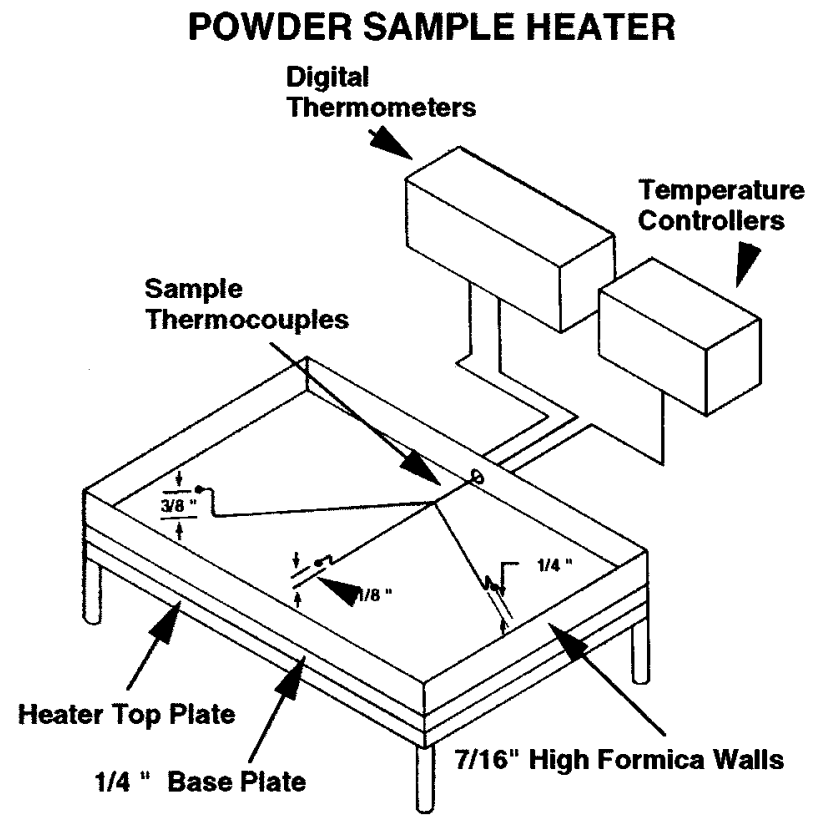

FIG. 1. Powder sample heater. The sample powders were loosely packed into this heater for measurements with the Network analyzer. The surfaces were smoothed off parallel to the bottom plate prior to measurements.

were found to be quite reproducible. Some of the experimental data show the reflection coefficient slightly above the zero line at some frequencies. [This would suggest a slight gain and is an artifact believed to be due to the powder sample surfaces not being perfectly flat and parallel to the back plane.]

\section{THEORY}

The centimeter-wave dielectric properties of sodium and potassium nitrates and nitrites are discussed in this section. The dielectric losses experienced in these materials are primarily the result of two fundamental loss mechanisms, one involving permanent dipole moments and the other involving dynamically induced dipole moments.

In sodium and potassium nitrite, the charges making up the nitrite ions are geometrically arranged in such a way as to produce a permanent dipole moment. ${ }^{1}$ At $300 \mathrm{~K}$ and above, it is believed that the nitrite ions in potassium nitrite are dynamically disordered and undergo quasi-free rotation. An external field will couple directly to the dipole moment, drive the rotations, and cause a weak, temperature-dependent dielectric loss. ${ }^{7}$ For sodium nitrite, the nitrite ions are ordered below $440 \mathrm{~K}$ and show little microwave activity. ${ }^{1}$ As the temperature rises towards $440 \mathrm{~K}$, the nitrite ions initiate a $180^{\circ}$ hopping motion parallel to the oxygen-oxygen direction and thus begin to couple strongly to the microwave field. As the temperature is raised to $440 \mathrm{~K}$ and above, the nitrite ions go collectively into quasi-free rotations and produce a dielectric response qualitatively similar to potassium nitrite. ${ }^{8}$

The nitrates show a more subtle behavior than the nitrites. The nitrate ion has no permanent dipole moment since its charges lie on the vertices of an equilateral triangle. ${ }^{1}$ Thus, the nitrate ions in sodium nitrate do not, to lowest order, couple to a microwave field when they begin to rotate.
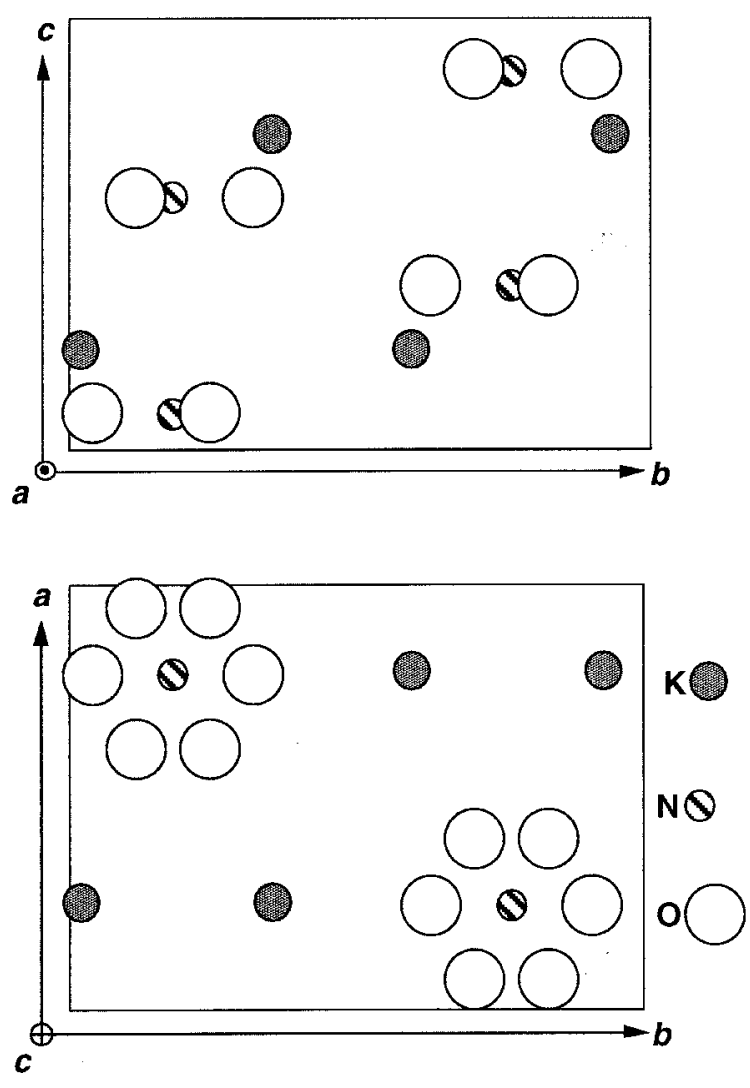

FIG. 2. Phase II representation of potassium nitrate lattice. The top figure shows projections of potassium nitrate ions onto the cb plane. Note the position of the $\mathrm{K}^{+}$ions above or below the planes of potassium nitrate. The bottom figure shows the potassium nitrate lattice looking along the $\mathbf{c}$ axis.

Also, the sodium ions lie exactly between the centroids of the nitrate ion triangles and therefore are largely unaffected by the nitrate ion rotations.

In potassium nitrate, the situation is more complex. From room temperature up to about $128^{\circ} \mathrm{C}$, it exists in an orthorhombic structure known as Phase II. If heated to above $128^{\circ} \mathrm{C}$, it undergoes a order/disorder transition to a hightemperature dynamically disordered trigonal structure designated as Phase I. This transition is accompanied by a large expansion along the $\mathbf{c}$ axis. Upon cooling from the hightemperature phase, potassium nitrate undergoes a transition into a metastable ferroelectric phase, Phase III. This phase exists from $\sim 124$ to $\sim 110^{\circ} \mathrm{C}$, below which a transition back to Phase II takes place.

Figure 2 shows two orthogonal projections of a room temperature, Phase II representation of the potassium nitrate lattice. When potassium nitrate is in this stable phase, the structure is orthorhombic $P m c n^{1,5,6}$ with the $\mathrm{K}^{+}$ions lying between the planar $\mathrm{NO}_{3}^{-}$ions along the $\mathbf{c}$ axis, but displaced along the $\mathbf{b}$ axis so they do not lie above or below the centroids of the $\mathrm{NO}_{3}^{-}$ions, as shown. In this phase, there is no net static dipole moment. That this phase is nonpolar results from the fact that, although there are dipoles associated with the $\mathrm{K}-\mathrm{NO}_{3}^{-}$chains along the $\mathbf{c}$ axis, these occur in antiparallel pairs producing zero net moment. However, when the 
temperature rises, the $\mathrm{NO}_{3}^{-}$ions are thermally activated into hindered rotation, and polar fluctuations occur in each chain. These do not cancel in pairs being, at best, only loosely correlated. It is these polar fluctuations that cause the dielectric losses that are observed up to about $128^{\circ} \mathrm{C}$. If the system is heated to above $128{ }^{\circ} \mathrm{C}$ into the high-temperature phase, Phase I, there is a radical restructuring involving: (a) the disordering of the $\mathrm{NO}_{3}^{-}$ions by hindered, almost free rotation about the $\mathbf{c}$ axis, and (b) a large displacement of the $\mathrm{K}^{+}$ ions within the ab plane such that they move to a position immediately below the centroids of the $\mathrm{NO}_{3}^{-}$ions, thus causing the large $\mathbf{c}$ axis expansion of about 5\%. In this hightemperature phase, since the $\mathrm{NO}_{3}^{-}$ions are in almost free rotation, they thus produce sixfold rather than threefold rotational symmetry about the $\mathbf{c}$ axis. They also then move to the midpoint of the adjacent $\mathrm{K}^{+}$planes (or vice versa). This motion should cause the polarity to vanish. However, this is only true on average, and about this average configuration there will be continuing fluctuations. This motion is polar and will couple to an external electric field-hence, the observed dielectric absorption in Phase I.

If now the crystal is cooled below $\sim 128{ }^{\circ} \mathrm{C}$, it transforms to a new phase, Phase III. In this ferroelectric phase, the $\mathrm{NO}_{3}^{-}$ions are now aligned so that they are all superposed when viewed down the $\mathbf{c}$ axis, but the $\mathrm{K}^{+}$ions do not lie halfway between them, being displaced from the midpoint by a finite distance. The $\mathrm{K}^{+}$ions remain located immediately above or below the $\mathrm{NO}_{3}^{-}$ion centroids. This causes the development of a spontaneous dipole moment along $\mathbf{c}$, which may be switched by reversing the displacement of the $\mathrm{K}^{+}$ ions to the other energetically equivalent structure. This implies a "double well" potential energy surface for the lattice, qualitatively similar to that in $\mathrm{BaTiO}_{3} \cdot{ }^{9-11}$ In both the ferroelectric phase and in Phase II (300 K, Stable), the basic impediment to full but hindered rotation of the $\mathrm{NO}_{3}^{-}$molecular ions is the presence of the layers of $\mathrm{K}^{+}$ions.

\section{RESULTS AND DISCUSSION}

Reflection measurements for " $50 \%$ dense powder" sodium nitrate at frequencies from 2 to $20 \mathrm{GHz}$ and at temperatures up to $240{ }^{\circ} \mathrm{C}$ are shown in Fig. 3. The losses are small even as the sample is heated above the order/disorder transition temperature. This is as expected because of the high symmetry of this system and the lack of a dynamically induced dipolar loss mechanism. These results are similar to those obtained for the ionic salts potassium bromide and sodium chloride. ${ }^{4}$

Figures 4-7 show reflectometer data and theoretical fits based on a Debye relaxation model for potassium nitrite, sodium nitrite, and potassium nitrate. The experimental data for potassium and sodium nitrite are for $50 \%$ density powders that are more than $1 \mathrm{~cm}$ thick. The potassium nitrate results were obtained from an almost fully dense compacted powder sample with a thickness of $8.1 \mathrm{~mm}$. The theoretical fits were obtained from a Debye form ${ }^{12}$ for the complex dielectric constant as follows:

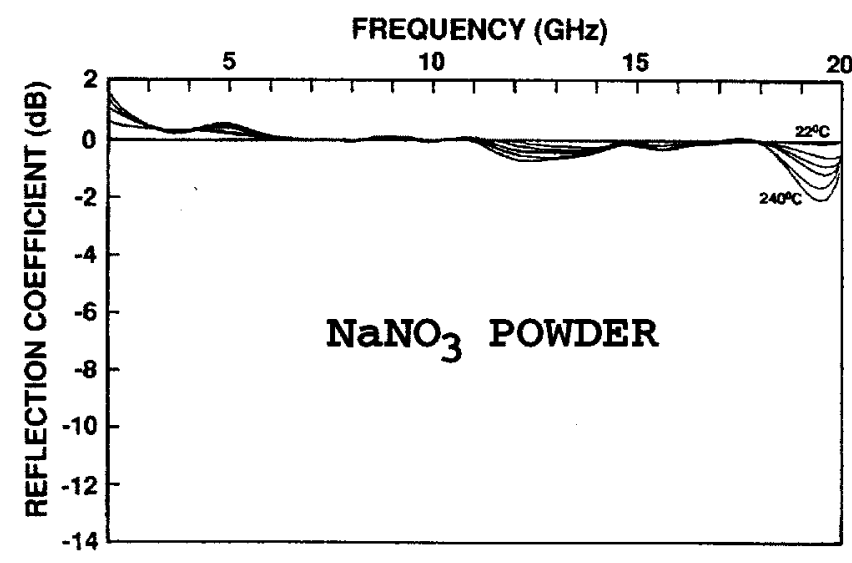

FIG. 3. Temperature-dependent microwave reflection curves for sodium nitrate powder sample.

$$
\begin{aligned}
& \epsilon=\epsilon_{\infty}+\frac{4 \pi a}{1+i 2 \pi v \tau}=\epsilon^{\prime}+i \epsilon^{\prime \prime}, \\
& \epsilon^{\prime}=\epsilon_{\infty}+\frac{4 \pi a}{1+4 \pi^{2} v^{2} \tau^{2}}, \\
& \epsilon^{\prime \prime}=\frac{8 \pi^{2} a v \tau}{1+4 \pi^{2} v^{2} \tau^{2}},
\end{aligned}
$$

where $\epsilon_{\infty}$ is the high-frequency dielectric constant, $a$ is the temperature-dependent parameter, $v$ is the frequency of incident radiation, $\tau$ is the relaxation time, and where $\tau=$ and $a=$ were adjusted to produce the best overall fit to the experimental absorption/reflectivity data.

Figure 4(a) shows the results of measurements on a 1.3$\mathrm{cm}$-thick, loosely packed powder of potassium nitrite at temperatures from 46 to $114{ }^{\circ} \mathrm{C}$. Significant losses occur above room temperature through $114{ }^{\circ} \mathrm{C}$ with a weak temperature variation. This Debye-like temperature dependence is in agreement with what is expected for this type of a polar solid. Figure 4(b) shows the theoretical fits to the experimentally obtained temperature data for potassium nitrite. The parameters chosen for the fits are shown in Table I. The value of $\epsilon_{\infty}=2.2$. was chosen as a reasonable value for less than full density loosely packed powder of potassium nitrite. Although some smaller features of the experimental data are not reproduced in the fits, generally the fits are quite good with the chosen parameters.

Figure 5(a) shows experimental data for sodium nitrite from room temperature to above $150{ }^{\circ} \mathrm{C}$. Experimentally, below $70{ }^{\circ} \mathrm{C}$, there is little change from the base line measurements. Above $100{ }^{\circ} \mathrm{C}$, the losses begin to develop and increase dramatically up to above $150{ }^{\circ} \mathrm{C}$. This implies that some thermally activated hopping of the nitrite ions occurs below the transition temperature of $440 \mathrm{~K}\left(167^{\circ} \mathrm{C}\right)$. Figure 5(b) shows the theoretical fits to the sodium nitrite data. The parameters used for the fits are shown in Table II. Although the strengths of some of the reflection minima are not perfectly represented in the theoretical fits, the fits obtained with a single parameter $(a=0.032$ to 0.001$)$ represent the data fairly well over the temperature range measured. 

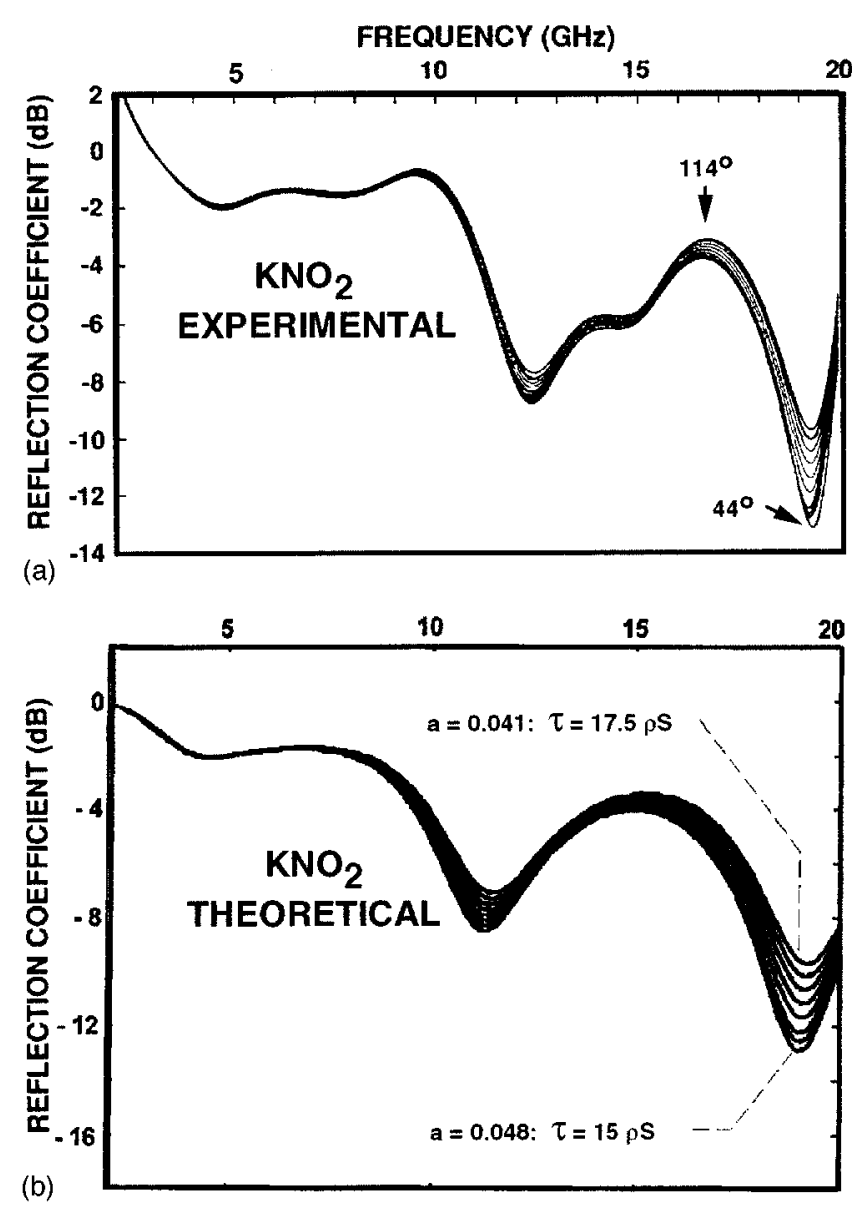

FIG. 4. Experimental reflectometer measurements and theoretical calculations for potassium nitrite at various temperatures.

Results of experimental measurements and theoretical fits on an almost fully dense, 8.1-mm-thick, pressed powder sample of potassium nitrate are shown in Fig. 6 and 7. The temperature was raised from room temperature to about $136{ }^{\circ} \mathrm{C}$. No attempt was made to measure the sample in the ferroelectric phase (Phase III) upon cooling from the hightemperature phase. In Fig. 6(a), experimental data are shown from room temperature to $116^{\circ}$. In Fig. 6(b), corresponding theoretical fits are shown. Table III shows the parameters utilized to obtain the fits for both Figs. 6 and 7. In Fig. 7(a), experimental data from 118 to $136^{\circ} \mathrm{C}$ are shown with the

TABLE I. Theoretical fits to $\mathrm{KNO}_{2}$ data. $^{\mathrm{a}}$

\begin{tabular}{ccc}
\hline \hline $\begin{array}{c}\text { Curve } \\
(\epsilon=2.2)\end{array}$ & $\mathrm{a}$ & $\begin{array}{c}\tau \\
\text { Relaxation time } \\
(\mathrm{pS})\end{array}$ \\
\hline Top & 0.041 & 17.5 \\
& 0.042 & 17 \\
& 0.043 & 16.5 \\
& 0.044 & 16 \\
& 0.045 & 15.5 \\
& 0.046 & 15 \\
Bottom & 0.047 & 15 \\
& 0.048 & 15 \\
\hline \hline
\end{tabular}

a Parameters used for Debye model calculations of the reflection coefficient. Thickness used $=1.3 \mathrm{~cm}$.

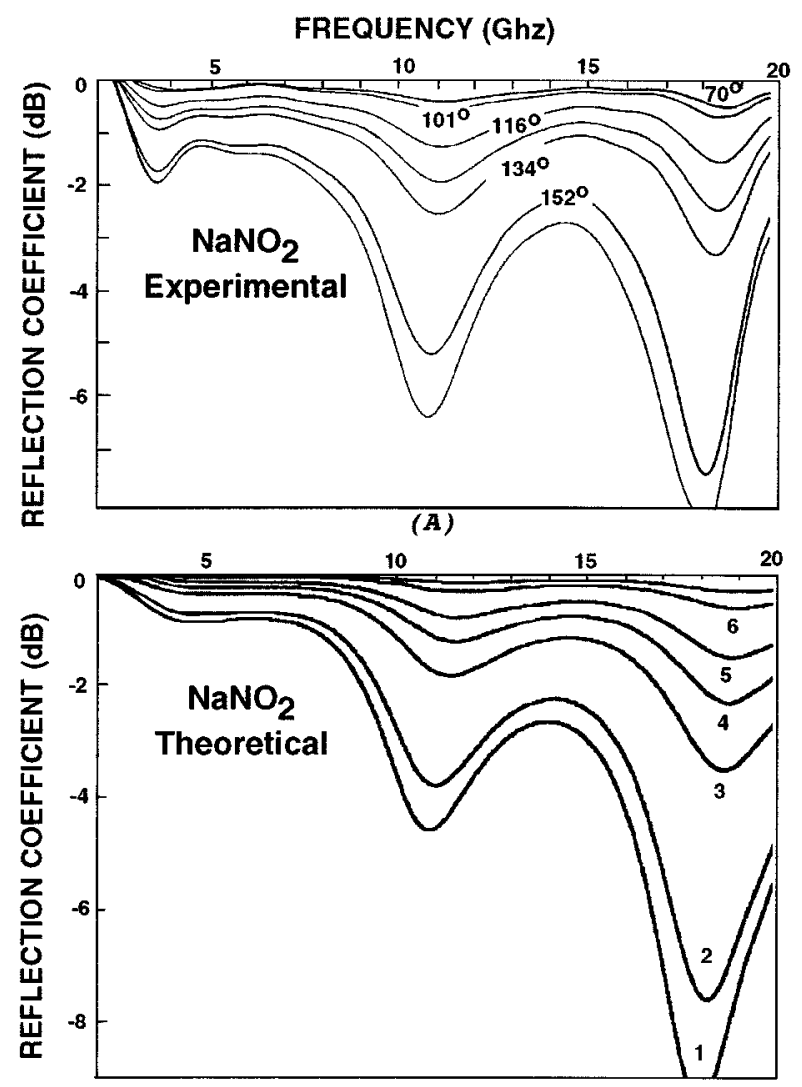

(B)

FIG. 5. Experimental reflectometer measurements and theoretical calculations for sodium nitrite at various temperatures.

corresponding theoretical fits shown in Fig. 7(b). As can be seen in the figures, reflection losses begin to occur as the temperature is raised to and above $100{ }^{\circ} \mathrm{C}$. Above this temperature, the two large reflection minima move to lower frequencies and become weaker. A smaller, low-frequency minimum (below $5 \mathrm{GHz}$ ) continues to grow as the temperature is raised to above $136{ }^{\circ} \mathrm{C}$. The results on potassium nitrate are strong evidence for the development of a dipolar loss mechanism above about $100{ }^{\circ} \mathrm{C}$ as discussed previously. The weakening of the losses above the transition can be explained by the movement of the potassium ions toward the centroid line joining the nitrate ions. This results in a de-

TABLE II. Theoretical fits to $\mathrm{NaNO}_{2}$ data. ${ }^{\mathrm{a}}$

\begin{tabular}{ccc}
\hline \hline $\begin{array}{c}\text { Curve } \\
(\epsilon=1.5)\end{array}$ & $\mathrm{a}$ & $\begin{array}{c}\tau \\
\text { Relaxation time } \\
(\mathrm{pS})\end{array}$ \\
\hline 1 & 0.032 & 8 \\
2 & 0.026 & 8 \\
3 & 0.012 & 8 \\
4 & 0.0078 & 8 \\
5 & 0.005 & 8 \\
6 & 0.002 & 8 \\
7 & 0.001 & 8 \\
\hline \hline
\end{tabular}

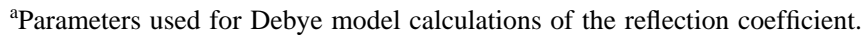
Thickness used $=1.35 \mathrm{~cm}$. 
FREQUENCY (GHz)
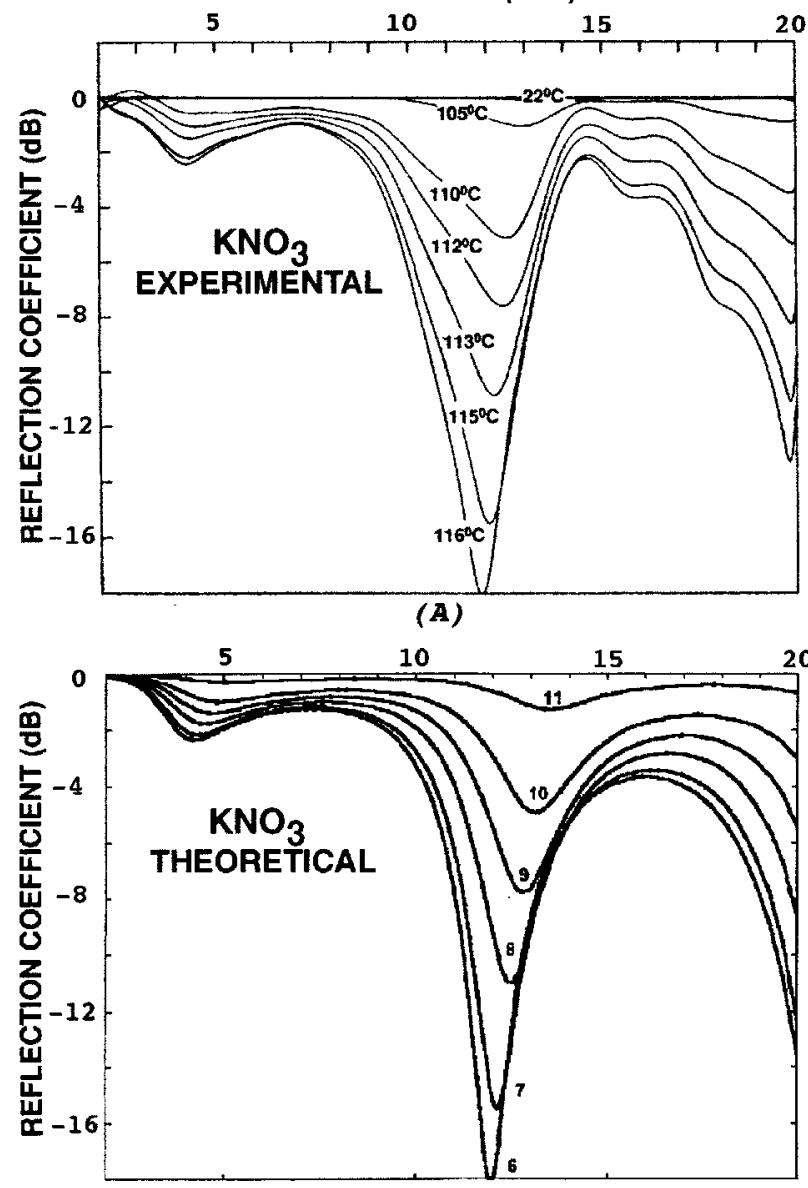

(B)

FIG. 6. Experimental reflectometer measurements and theoretical results from room temperature to $116^{\circ} \mathrm{C}$ for nearly fully dense pressed powder potassium nitrate.

crease in the dynamically induced dipole moment and thus the mechanism that leads to loss in this material.

When one compares theory with experiment, it is evident that the agreement with experiment is remarkably close given that only two parameters are employed. Indeed, one can obtain fits almost as good by varying the parameter " $a$ ",

TABLE III. Theoretical fits to $\mathrm{KNO}_{3}$ data. ${ }^{\mathrm{a}}$

\begin{tabular}{rlc}
\hline \hline $\begin{array}{c}\text { Curve } \\
(\epsilon=2.2)\end{array}$ & \multicolumn{1}{c}{$\begin{array}{c}\tau \\
\text { Relaxation time } \\
(\mathrm{pS})\end{array}$} \\
\hline 1 & 0.39 & 6 \\
2 & 0.33 & 6.8 \\
3 & 0.28 & 7.08 \\
4 & 0.24 & 7.1 \\
5 & 0.2 & 8.2 \\
6 & 0.135 & 9 \\
7 & 0.12 & 9 \\
8 & 0.09 & 9 \\
9 & 0.064 & 9.3 \\
10 & 0.04 & 9.6 \\
11 & 0.01 & 10 \\
\hline \hline
\end{tabular}

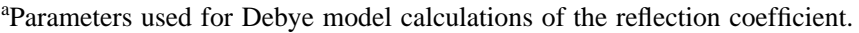
Thickness used $=8.2 \mathrm{~mm}$.
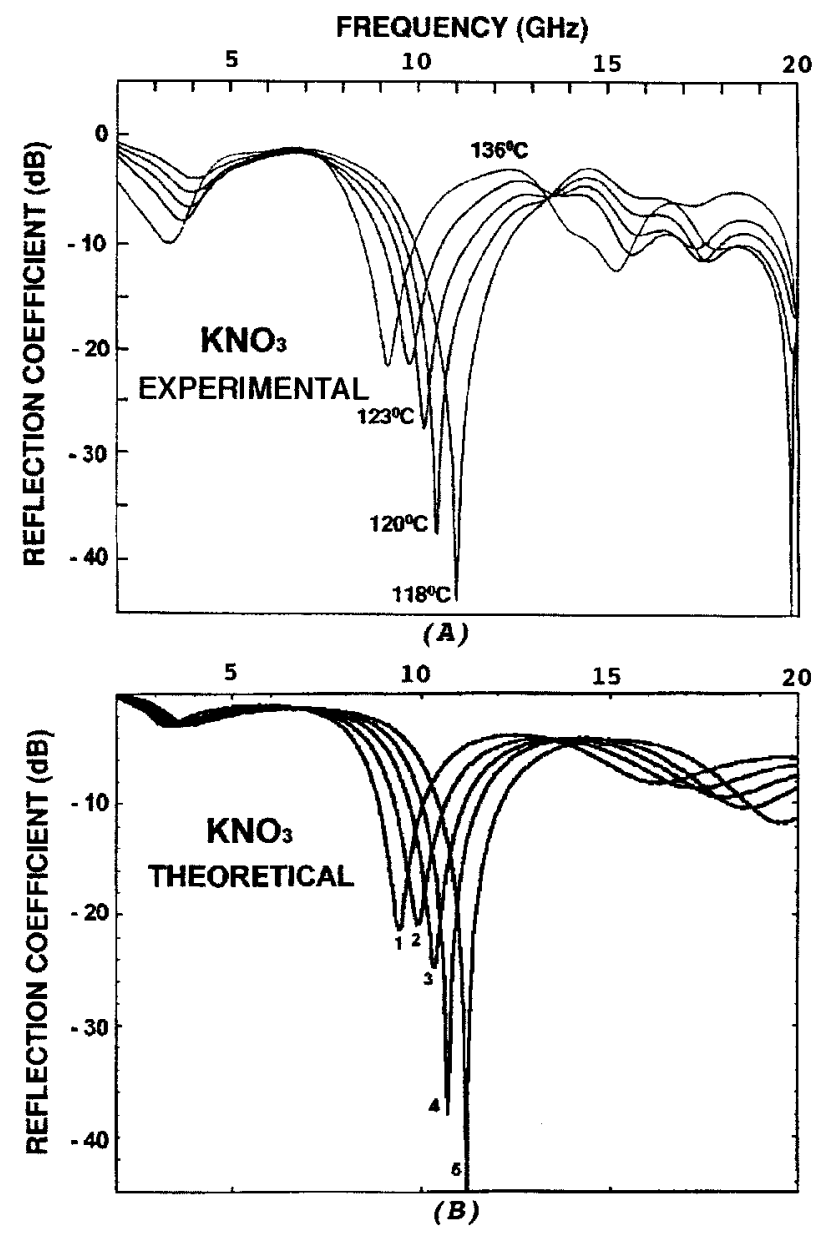

FIG. 7. Experimental reflectometer measurements and theoretical results from 118 to $136{ }^{\circ} \mathrm{C}$ for nearly fully dense pressed powder potassium nitrate.

alone. However, the modest variations in $\tau$ that have been employed are consistent with the $(T)^{-1}$ dependence that one expects from high-temperature phonon damping.

More germane to the theoretical fits to experiment is the derivation of the values of $a$ from basic principles. The simplest possible approach is to assume that these losses are due to arrays of independent dipoles whose moments can be derived or inferred from the structural data. The resultant expression for the coefficient $a$ is

$$
a=\frac{N \eta^{2}}{K T},
$$

where $N$ is the number of dipoles/unit volume, $\eta$ is the magnitude of this moment, $K$ is Boltzmann's constant, and $T$ is the temperature. ${ }^{12}$

For the potassium nitrate dipole, we can approximate $\eta$ by the polarization per unit cell in the metastable ferroelectric phase (Phase III) obtained from the observed and theoretical values of $\simeq 10 \mu \mathrm{C} / \mathrm{cm}^{2} .{ }^{13}$

If we apply the aforementioned values, we find that the theoretical value of $a \approx 1.5$ giving $\epsilon(\omega \rightarrow 0) \approx 20$ for a field parallel to the polar axis of Phase I with the use of $\epsilon_{\infty}=4.2$. However, Chen and Chernow ${ }^{13}$ report a value for $\epsilon(\omega \rightarrow 0)$ $\approx 50-60$ for Phase I, when the electric field is applied along 
the $\mathbf{c}$ axis. This difference is not a serious problem since our independent dipole model neglects two significant effects, both of which will enhance $\epsilon \| \mathbf{c}$ : (a) any correlation between dipoles (e.g., a mean field term), and (b) allowance for local field effects.

However, there is a major problem with our experimentally fitted values of $a$. They are too small to be consistent with the data of Chen and Chernow. ${ }^{3}$ Since our specimens are pressed powders, and we only expect $\epsilon \| \mathbf{c}$ to show these much enhanced values, then our experimental value of $\epsilon(\omega \rightarrow 0)$ should be approximately $1 / 3$ of their value of $\epsilon(\omega \rightarrow 0)$ when the field is parallel $\mathbf{c}$ (i.e., $\sim 20)$. While this only requires the fitted value of $a \approx 1.5$, this is almost triple the largest value obtained from the fits to the experimental data.

Clearly, we have a major discrepancy, and the most likely explanation is nonuniformity of temperature across the specimens that are approximately $1 \mathrm{~cm}$ thick. Since the transition is very sharp and jumps discontinuously from $\sim 10$ to $\sim 50-60$ at transition, it appears likely there will be a sharp interface between the transformed and the untransformed material that will travel from the metal backing to the surface as the former is heated. Thus, the absorption that is actually measured is loss averaged over the specimen thickness, with most of the loss probably coming from a thin layer whose temperature is at or very close to $T_{c}$. Thus, the effective thickness is much less than the actual width.

In the case of the nitrites, sodium nitrite shows analogous behavior to potassium nitrate except that the nature of the dipoles is different, as we have discussed previously. However, it appears that none of the specimen has actually undergone transformation into the high-temperature phase.

\section{SUMMARY}

We have studied the temperature-dependent centimeterwave reflection properties of powdered samples of several molecular crystals. The results of reflectometer temperaturedependent measurements were compared to theoretical calculations based on a Debye relaxation model for the potassium nitrate, potassium nitrite, and sodium nitrite samples. The nitrite samples showed absorptions when temperatures were high enough to excite hopping modes. For potassium nitrite, losses were seen at all of the measured temperatures, and sodium nitrite showed losses above about $100{ }^{\circ} \mathrm{C}$ below the $165{ }^{\circ} \mathrm{C}$ transition temperature. Sodium nitrate showed little losses, but potassium nitrate showed interesting behavior around or above the order/disorder transition temperature of about $128{ }^{\circ} \mathrm{C}$. This behavior is very likely caused by a dynamically induced dipolar loss mechanism.

\section{ACKNOWLEDGMENT}

The authors would like to thank Dr. Robert Bossoli for his expertise with the network analyzer and his help with the experimental measurements.

${ }^{1}$ H. D. Megaw, Crystal Structures, A Working Approach (Saunders, Philadelphia, 1973).

${ }^{2}$ A. R. Von Hippel, Dielectric Materials and Applications (Massachusetts Institute of Technology Press, Cambridge, MA, 1954).

${ }^{3}$ F. Jona and G. Shirane, Ferroelectric Crystals (Macmillan, New York, 1962).

${ }^{4}$ S. G. Cornelison, A. Gauss, Jr., R. B. Bossoli, H. M. Lu, and J. R. Hardy, BRI-MR-4002, U.S. Army Ballistic Research Laboratory, Aberdeen Proving Ground, MD, September 1992.

${ }^{5}$ H. M. Lu and J. R. Hardy, Ferroelectrics 111, 43 (1990)

${ }^{6}$ H. M. Lu and J. R. Hardy, Phys. Rev. B 44, 7215 (1991).

${ }^{7}$ D. M. Adams, M. Pogson, and S. K. Sharma, J. Phys. C 21, 623 (1988).

${ }^{8}$ H. M. Lu, R. Qi, and J. R. Hardy, Solid State Commun. 87, 1151 (1993).

${ }^{9}$ R. E. Cohen and H. Krakauer, Phys. Rev. B 42, 6416 (1990).

${ }^{10}$ R. E. Cohen and H. Krakauer, Ferroelectrics 136, 65 (1992).

${ }^{11}$ R. E. Cohen, Nature (London) 358, 136 (1992).

${ }^{12}$ C. Kittle, Introduction to Solid State Physics, 1st ed. (Wiley, New York, 1953), p. 89.

${ }^{13}$ A. Chen and F. Chernow, Phys. Rev. 154, 493 (1967). 